\title{
Periprocedural Complications in Patients Undergoing Catheter Ablation of Atrial Fibrillation Without Discontinuation of a Vitamin K Antagonist and Direct Oral Anticoagulants
}

\author{
Toshiya Kojima, MD, PhD; Katsuhito Fujiu, MD, PhD; Nobuaki Fukuma, MD; \\ Hiroshi Matsunaga, MD; Tsukasa Oshima, MD; Jun Matsuda, MD; \\ Takumi Matsubara, MD; Yu Shimizu, MD, PhD; Gaku Oguri, MD, PhD; \\ Eriko Hasumi, MD, PhD; Hiroyuki Morita, MD, PhD; Issei Komuro, MD, PhD
}

\begin{abstract}
Background: Periprocedural anticoagulation is important in catheter ablation (CA) of atrial fibrillation (AF) and there is increasing evidence that uninterrupted vitamin K antagonist (VKA) therapy is superior to interrupted anticoagulation strategies. Since the emergence of direct oral anticoagulants (DOACs), numerous studies have shown promising results for their use in uninterrupted strategies. However, further studies are needed to further define the efficacy and safety of performing AF ablation with uninterrupted factor XA inhibitors or direct thrombin inhibitors.

Methods and Results: We have performed CA of AF without discontinuation of either VKA or DOAC therapy since April 2014. A total of 376 patients with AF underwent CA including pulmonary vein isolation. All of the patients were divided into 2 groups (uninterrupted VKA or uninterrupted DOACs). Anticoagulation with DOACs was associated with fewer complications than uninterrupted VKA therapy $(P=0.04)$. There were significant differences between groups in the rates of congestive heart failure, left ventricular ejection fraction, body weight, and estimated glomerular filtration rate and of the $\mathrm{CHADS}_{2}, \mathrm{CHA}_{2} \mathrm{DS}_{2}-\mathrm{VASc}$ and HAS-BLED scores. Therefore, we also analyzed the results using the propensity score-matching method. We found no significant difference in periprocedural complications between uninterrupted VKA or DOACs therapy $(P=0.65)$.
\end{abstract}

Conclusions: CA of AF without discontinuation of DOACs is not inferior to CA without discontinuation of a VKA, with regard to ischemic or hemorrhagic complications.

Key Words: Ablation; Anticoagulants; Atrial fibrillation

$\mathbf{P}$ eriprocedural anticoagulation is important for successful catheter ablation (CA) of atrial fibrillation (AF). The most significant complications associated with ablation of AF are periprocedural stroke/transient ischemic attack (TIA), bleeding, and cardiac tamponade, ${ }^{1}$ and anticoagulation is important for reducing the risk of cerebrovascular events. ${ }^{1}$ There is increasing evidence that uninterrupted vitamin $\mathrm{K}$ antagonist (VKA) therapy is superior to interrupted anticoagulation strategies. Di Biase et al showed that performing $\mathrm{CA}$ of $\mathrm{AF}$ without discontinuation of warfarin significantly reduced the occurrence of periprocedural stroke/TIA and bleeding complications., 2,3 Since the emergence of direct oral anticoagulants (DOACs), numerous studies have shown promising results when using them in uninterrupted strategies. Calkins et al showed anticoagulation with uninterrupted dabigatran was associated with fewer bleeding complications than with uninterrupted warfarin in the RE-CIRCUIT trials. ${ }^{4}$ Some preliminary data are available from observational studies of uninterrupted DOAC strategies. ${ }^{\mathbf{5}, 6}$ In the 2017 HRS/ EHRA/ECAS/APHRS/SOLAECE expert consensus statement on catheter and surgical ablation of AF, a strategy of performing AF ablation in patients receiving uninterrupted anticoagulation is considered to be safe and minimizes the risk of thromboembolic events. However, it is also noted that further studies are needed to further define the efficacy and safety of performing AF ablation with uninterrupted factor XA inhibitors or direct thrombin inhibitors. ${ }^{\mathbf{7}, 8}$ The technique of uninterrupted anticoagulation is becoming more widespread, but in many institutions, DOACs are still interrupted before CA. There are 4 DOACs, with differing oral administration regimens: dabigatran and apixaban

Received October 10, 2017; revised manuscript received February 14, 2018; accepted February 21, 2018; released online April 13, 2018 Time for primary review: 12 days

Department of Cardiovascular Medicine (T.K., K.F., N.F., H. Matsunaga, T.O., J.M., T.M., Y.S., G.O., E.H., H. Morita, I.K.), Department of Ubiquitous Health Informatics (K.F.), Clinical Research Support Center (G.O.), The University of Tokyo, Tokyo, Japan

Mailing address: Toshiya Kojima, MD, PhD, Department of Cardiovascular Medicine, The University of Tokyo Graduate School of Medicine, 7-3-1 Hongo, Bunkyo-ku, Tokyo 113-8655, Japan. E-mail: toshiyakojima-circ@umin.ac.jp

ISSN-1346-9843 All rights are reserved to the Japanese Circulation Society. For permissions, please e-mail: cj@j-circ.or.jp 


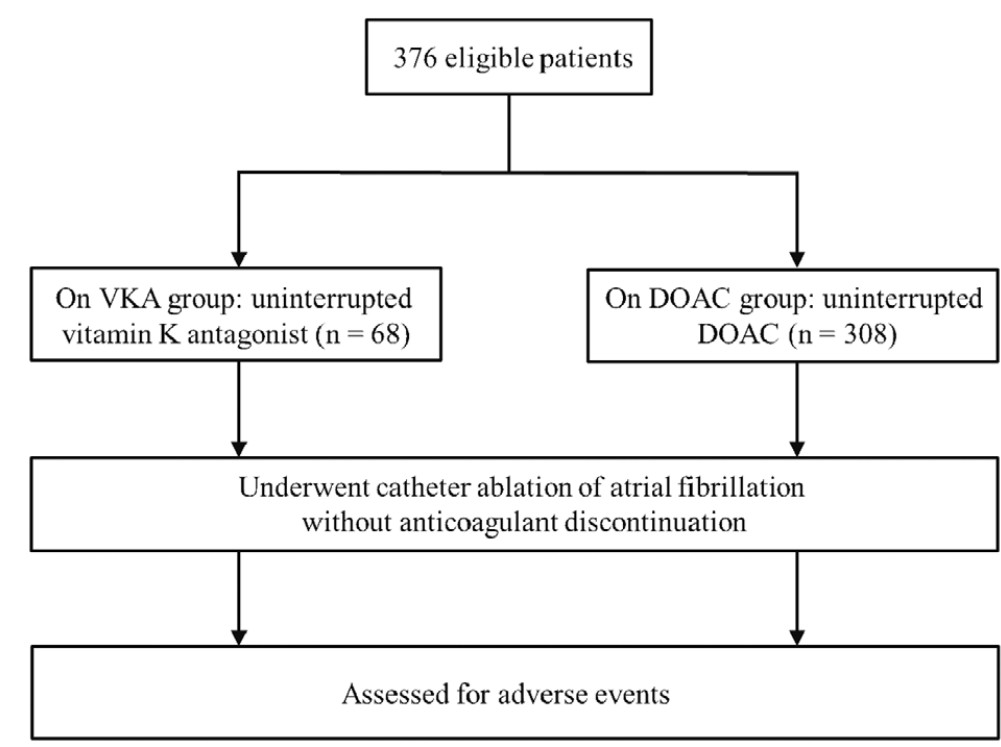

Figure. Study design, enrollment, and followup. DOAC, direct oral anticoagulant; VKA, vitamin $\mathrm{K}$ antagonist.

\begin{tabular}{|ccccc|}
\hline Table 1. Anticoagulants & & & \\
VKA & Dabigatran & Ribaroxaban & Apixaban & Edoxaban \\
68 & 41 & 117 & 105 & 45 \\
\hline
\end{tabular}

DOAC, direct oral anticoagulant; VKA, vitamin $\mathrm{K}$ antagonist.

require twice-daily dosing; rivaroxaban and edoxaban are once-daily dosing.

\section{Methods}

We have performed CA of AF without discontinuation of VKA or DOACs since April 2014, so we conducted a retrospective, observational, single-center study to assess this technique. We excluded hemodialysis cases, because of the significant high-risk population. All components of standard informed consent, including the purpose of the study, risk, and benefits, were fully explained to the patients before enrollment. The study protocol conformed to the Declaration of Helsinki and was reviewed and approved by the Institutional Review Board of the University of Tokyo, Tokyo, Japan (no. 2650).

\section{Anticoagulant Management}

All patients were on anticoagulants for longer than 4 weeks before CA. Preprocedural transesophageal echocardiography was performed to confirm the absence of thrombus. All patients continued anticoagulant therapy uninterruptedly. In the VKA group, the patients' international normalized ratio (INR) was between 1.5 and 3 . A bolus of 5,000 IU heparin was administered before transseptal puncture and a continuous infusion of heparin $1,000 \mathrm{U} / \mathrm{h}$ was started. During the procedure, the activated clotting time was maintained at 300-350 s. ${ }^{9}$ After ablation was completed, protamine sulfate was administered to reverse the effect of heparin. After each procedure, anticoagulants were administered for at least 4 weeks as per the patient's schedule prior to ablation. There was no significant difference in periproce- dural anticoagulant management in either treatment group.

\section{Ablation Procedure}

Extensive encircling pulmonary vein isolation (PVI) guided by a mapping catheter and intracardiac echocardiography was performed. All PVI procedures were performed by the same experienced operators (>500 PVIs). If PVI was insufficient to sustain sinus rhythm, linear ablation (mitral isthmus linear ablation, left atrium (LA) roof linear ablation, or LA anterior linear ablation), ablation of complex fractionated atrial electrocardiograms, ganglionated plexi ablation, and/ or superior vena cava isolation was added. In patients with persistent/long-standing persistent AF (PeAF/LSAF), if AF was not terminated, electric cardioversion was performed to restore sinus rhythm. ${ }^{10}$ Adenosine triphosphate and isoproterenol were usually administered to disclose dormant conduction of PV and non-PV triggers.

\section{Adverse Events}

All adverse events throughout the periprocedural period and up to the time of the first outpatient visit were recorded and analyzed. Adverse events included TIA, bleeding, and cardiac tamponade. Bleeding usually includes major bleeding, defined as intracerebral hemorrhage, and bleeding requiring intervention, causing symptoms, or requiring transfusion. Minor bleeding is defined as the occurrence of hematoma or any bleeding that does not require any intervention or did not cause any symptoms.11,12 However, in this study, there were no cases of major bleeding, so we decided to collate all bleeding events. 


\begin{tabular}{|c|c|c|c|}
\hline & $\begin{array}{c}\text { VKA } \\
(n=68)\end{array}$ & $\begin{array}{c}\text { DOAC } \\
(n=308)\end{array}$ & $P$ value \\
\hline Male, n (\%) & $48(71)$ & $224(73)$ & 0.72 \\
\hline Age (years) & 65.4 & 63.9 & 0.31 \\
\hline \multicolumn{4}{|l|}{ AF type, n (\%) } \\
\hline PAF & $29(43)$ & $157(51)$ & 0.21 \\
\hline PeAF & $30(44)$ & $118(38)$ & 0.38 \\
\hline LSAF & $9(13)$ & $33(11)$ & 0.55 \\
\hline CHF, n (\%) & $34(50)$ & $54(18)$ & $<0.01$ \\
\hline HT, n (\%) & $39(57)$ & $172(56)$ & 0.82 \\
\hline DM, n (\%) & $14(21)$ & $50(16)$ & 0.39 \\
\hline Prior stroke/TIA, n (\%) & $7(10)$ & $32(10)$ & 0.98 \\
\hline LVEF (\%) & 54 & 62 & $<0.01$ \\
\hline LAd (mm) & 42 & 41 & 0.14 \\
\hline BW (kg) & 63.9 & 66.2 & 0.19 \\
\hline eGFR (mL/min/1.73 m²) & 56.1 & 66.8 & $<0.01$ \\
\hline CHADS$_{2}$ score, n (\%) & 1.72 & 1.28 & $<0.01$ \\
\hline 0 & $11(16)$ & $80(26)$ & \\
\hline 1 & $17(25)$ & $120(39)$ & \\
\hline 2 & $24(35)$ & $65(21)$ & \\
\hline 3 & $12(18)$ & $30(10)$ & \\
\hline 4 & $4(6)$ & $11(4)$ & \\
\hline 5 & $0(0)$ & $2(1)$ & \\
\hline $\mathrm{CHA}_{2} \mathrm{DS}_{2}$-VASc score, $\mathrm{n}(\%)$ & 2.95 & 2.08 & $<0.01$ \\
\hline 0 & $3(4)$ & $58(19)$ & \\
\hline 1 & $14(21)$ & $77(25)$ & \\
\hline 2 & $12(17)$ & $58(19)$ & \\
\hline 3 & $14(21)$ & $51(18)$ & \\
\hline 4 & $17(25)$ & $37(12)$ & \\
\hline 5 & $4(6)$ & $18(6)$ & \\
\hline 6 & $4(6)$ & $7(2)$ & \\
\hline 7 & $0(0)$ & $2(1)$ & \\
\hline 8 & $0(0)$ & $0(0)$ & \\
\hline 9 & $0(0)$ & $0(0)$ & \\
\hline HAS-BLED, n (\%) & 2.26 & 1.37 & $<0.01$ \\
\hline 0 & $7(10)$ & $74(24)$ & \\
\hline 1 & $18(26)$ & $105(34)$ & \\
\hline 2 & $25(37)$ & $84(27)$ & \\
\hline 3 & $9(13)$ & $33(11)$ & \\
\hline 4 & $3(5)$ & $11(4)$ & \\
\hline 5 & $6(9)$ & $1(0)$ & \\
\hline 6 & $0(0)$ & $0(0)$ & \\
\hline 7 & $0(0)$ & $0(0)$ & \\
\hline 8 & $0(0)$ & $0(0)$ & \\
\hline 9 & $0(0)$ & $0(0)$ & \\
\hline
\end{tabular}

$\mathrm{AF}$, atrial fibrillation (LS, longstanding; $\mathrm{P}$, paroxysmal; $\mathrm{Pe}$, persistent); $\mathrm{BW}$, body weight; $\mathrm{CHF}$, congestive heart failure; DM, diabetes mellitus; eGFR, estimated glomerular filtration rate; HT, hypertension; LAd, left atrial dimension; LVEF, left ventricular ejection fraction; TIA, transient ischemic attack. Other abbreviations as in Table 1.

\begin{tabular}{|lcrc|}
\hline \multicolumn{4}{l}{ Table 3. Complications Without Matching } \\
Stroke/TIA & VKA & DOAC & P value \\
Bleeding & $2(2.9)$ & $1(0.3)$ & 0.03 \\
Cardiac tamponade & $3(4.4)$ & $6(1.9)$ & 0.23 \\
Total & $1(1.5)$ & $3(1.0)$ & 0.72 \\
\hline
\end{tabular}

Abbreviations as in Tables 1,2.

\section{Statistical Analysis}

Continuous data are described as mean $\pm \mathrm{SD}$, and discrete data are described as n (\%). Student's t-test and the $\chi^{2}$ test (Fisher's exact test was used for cell values $<10$ ) were used to compare differences across groups. A multivariable logistic model was used to identify significant predictors of periprocedural adverse events. We used propensity score matching to determine differences between 2 groups in 


\begin{tabular}{|ccccc|}
\hline \multicolumn{2}{l}{ Table 4. Anticoagulants After Propensity Score Matching } & & \\
VKA & Dabigatran & Ribaroxaban & Apixaban & Edoxaban \\
63 & 7 & 24 & 22 & 10 \\
\hline
\end{tabular}

Abbreviations as in Table 1.

which the patient populations were too divergent to make meaningful comparisons. We created a logistic-regression model to derive propensity scores. The logistic regression of propensity for DOACs had an area under the receiveroperating characteristics curve of 0.79 , indicating good discrimination between patients managed with VKA and DOACs. The values for important variables associated with the use of DOACs included HAS-BLED score, estimated glomerular filtration rate (eGFR), left ventricular ejection fraction (LVEF), body weight $\left(\mathrm{BW}, \mathrm{kg}\right.$ ) and $\mathrm{CHADS}_{2}$ score. Analyses were performed with JMP Pro 13.0 (SAS Institute Inc., Cary, NC, USA).

\section{Results}

\section{Patients' Characteristics}

A total of 376 patients with AF underwent CA including PVI (Figure). All of the patients were divided into 2 groups (uninterrupted VKA or uninterrupted DOAC therapy). The types of anticoagulants are shown in Table 1 and the baseline characteristics and risk factors of the patients are shown in Table 2 . In the VKA group, 50\% had congestive heart failure $(\mathrm{CHF}), \mathrm{LVEF}$ was $54 \%$, and the average $\mathrm{CHADS}_{2}, \mathrm{CHA}_{2} \mathrm{DS}_{2}$-VASc and HAS-BLED score was $1.72,2.95$, and 2.26, respectively. In the DOAC group, $18 \%$ had CHF, LVEF was $62 \%$, and the average CHADS, $\mathrm{CHA}_{2} \mathrm{DS}_{2}-\mathrm{VASc}$ and HAS-BLED score was 1.28, 2.08, and 1.37 , respectively. We need to pay attention to the significant differences in the characteristics of the 2 groups. In VKA group, the average PT-INR was 2.05 and the average time in therapeutic range (TTR) was $65.9 \%$ just before the day of the procedure.

\section{Complications}

No fatal events were reported in either treatment group. Stroke/TIA occurred in $2(2.9 \%)$ patients in the VKA group and in $1(0.3 \%)$ patient in the DOAC group $(\mathrm{P}=0.03)$. Bleeding complications occurred in $3(4.4 \%)$ patients in the VKA group and in $6(1.9 \%)$ patients in the DOAC group $(\mathrm{P}=0.23)$. There was a significantly higher rate of total complications in the VKA group than in the DOAC group $(\mathrm{P}<0.04)$. Table 3 shows the adverse events; there were no significant differences in PT-INR and TTR between the groups with and without complications in the VKA group (PT-INR 2.05 vs. 2.11, $\mathrm{P}=0.80$; TTR 66.3 vs. $61.7, \mathrm{P}=0.82$ ). Stroke events did not occur in patients with significantly low PT-INR, and bleeding events did not occur in patients with significantly high PT-INR. In the DOAC group, only 1 patient with cerebral infarction had underdosing of dabigatran, and 1 patient with bleeding and cardiac tamponade events had underdosing of ribaroxaban. The other 8 patients had regular-dose DOACs. There were 4 cases of cardiac tamponade: 1 was VKA and the others were DOACs. All of them were recovered by epicardial drainage. Two patients received a blood infusion (Table S1).

However, as mentioned before, we could not estimate these results because there were significant differences in the baseline characteristics of the 2 groups.

Although it was a supplementary analysis because of the small sample size after matching, we used propensity score matching. The distribution of anticoagulants after propensity score matching is shown in Table 4 . The characteristics and risk factors after propensity score matching are shown in Table 5. There were no significant differences in the characteristics of the groups after propensity score matching. Total complications occurred in $3(4.8 \%)$ patients in the VKA group and in $2(3.2 \%)$ patients in the DOAC group $(\mathrm{P}=0.65)$. Collectively, even in the supplementary data, there were no significant differences between the groups for any of the complications (Table 6).

\section{Discussion}

This retrospective, observational study assessed complications of CA of AF with uninterrupted DOACs compared with uninterrupted VKA therapy. Given the data showing superiority or non-inferiority of less bleeding and incidence of stroke with DOACs compared with VKA therapy, more patients are being switched from VKA to DOACs. ${ }^{13}$ Previous clinical trials have shown that continuous VKA therapy is associated with fewer embolic events than interrupted treatment. ${ }^{1,2}$ However, data on the clinical outcomes of CA with continuous DOAC therapy are limited. ${ }^{6,14}$ Conflicting results comparing dabigatran with VKA therapy are present in the literature; ${ }^{15-17}$ however, the 2017 HRS/EHRA/ECAS/ APHRS/SOLAECE expert consensus statement on catheter and surgical ablation of $\mathrm{AF}^{\mathbf{7}, \mathbf{8}}$ considers that a strategy of performing AF ablation on patients receiving uninterrupted anticoagulation can be safe and minimizes the risk of thromboembolic events. Many of the observational and nonrandomized studies have had significant differences in the baseline characteristics of the study populations. In our study, there were significant differences in the rates of $\mathrm{CHF}$, LVEF, eGFR and of the $\mathrm{CHADS}_{2}, \mathrm{CHA}_{2} \mathrm{DS}_{2}-\mathrm{VASc}$, and HAS-BLED scores between the groups. The VKA group showed a disadvantage in all these characteristics. The DOAC group had a significantly lower rate of complications throughout the periprocedural period and up to the time of the first outpatient visit compared with the VKA group. All cases of cardiac tamponade were treated with pericardial drainage, because we believe that it is safer to manage with drainage as much as possible. In this study, there were no significant differences in the type of DOAC, the timing of oral administration (once or twice daily) or the timing of the procedure (AM or PM).

Although there was the limitation of the sample size becoming small, we used the propensity score-matching method to determine differences in clinical complications between the 2 groups in which the patients' characteristics were too divergent to make meaningful comparisons. After propensity score matching, the baseline characteristics and risk factors were well balanced between the groups (Table 5), and there were no significant differences in any complications between the groups (Table 6). Performing CA of AF without 


\begin{tabular}{|c|c|c|c|}
\hline & $\begin{array}{c}\text { VKA } \\
(n=63)\end{array}$ & $\begin{array}{l}\text { DOAC } \\
(n=63)\end{array}$ & $P$ value \\
\hline Male, n (\%) & $45(71)$ & $48(76)$ & 0.54 \\
\hline Age (years) & 65.0 & 65.1 & 0.97 \\
\hline \multicolumn{4}{|l|}{ AF type, n (\%) } \\
\hline PAF & $26(41)$ & $21(33)$ & 0.36 \\
\hline PeAF & $28(44)$ & $34(54)$ & 0.29 \\
\hline LSAF & $9(14)$ & $8(13)$ & 0.8 \\
\hline CHF, n (\%) & $23(37)$ & $17(27)$ & 0.25 \\
\hline HT, n (\%) & $36(57)$ & $42(67)$ & 0.27 \\
\hline DM, n (\%) & $11(17)$ & $13(21)$ & 0.65 \\
\hline Prior stroke/TIA, n (\%) & $7(11)$ & $8(13)$ & 0.79 \\
\hline LVEF & 54.5 & 54.7 & 0.94 \\
\hline LAD & 42.2 & 42.2 & 0.98 \\
\hline BW & 64.4 & 65.1 & 0.78 \\
\hline eGFR & 56.4 & 58.0 & 0.58 \\
\hline CHADS $_{2}$ score, $n(\%)$ & 1.70 & 1.59 & 0.58 \\
\hline 0 & $9(14)$ & $10(16)$ & \\
\hline 1 & $17(27)$ & $26(41)$ & \\
\hline 2 & $23(37)$ & $14(22)$ & \\
\hline 3 & $12(19)$ & $8(13)$ & \\
\hline 4 & $2(3)$ & $3(5)$ & \\
\hline 5 & $0(0)$ & $2(3)$ & \\
\hline $\mathrm{CHA}_{2} \mathrm{DS}_{2}$-VASc score, $\mathrm{n}(\%)$ & 2.76 & 2.62 & 0.63 \\
\hline 0 & $2(3)$ & $5(8)$ & \\
\hline 1 & $14(22)$ & $17(27)$ & \\
\hline 2 & $11(17)$ & $15(24)$ & \\
\hline 3 & $14(22)$ & $5(8)$ & \\
\hline 4 & $16(25)$ & $11(17)$ & \\
\hline 5 & $4(6)$ & $3(5)$ & \\
\hline 6 & $2(3)$ & $5(8)$ & \\
\hline 7 & $0(0)$ & $2(3)$ & \\
\hline 8 & $0(0)$ & $0(0)$ & \\
\hline 9 & $0(0)$ & $0(0)$ & \\
\hline HAS-BLED, n (\%) & 1.97 & 1.78 & 0.41 \\
\hline 0 & $7(11)$ & $10(16)$ & \\
\hline 1 & $16(25)$ & $19(30)$ & \\
\hline 2 & $25(40)$ & $17(27)$ & \\
\hline 3 & $7(11)$ & $10(16)$ & \\
\hline 4 & $3(5)$ & $6(10)$ & \\
\hline 5 & $5(8)$ & $1(2)$ & \\
\hline 6 & $0(0)$ & $0(0)$ & \\
\hline 7 & $0(0)$ & $0(0)$ & \\
\hline 8 & $0(0)$ & $0(0)$ & \\
\hline 9 & $0(0)$ & $0(0)$ & \\
\hline
\end{tabular}

Abbreviations as in Tables 1,2.

Table 6. Complications After Propensity Score Matching

\begin{tabular}{lccc} 
& VKA & DOAC & P value \\
Stroke/TIA & $2(3.2)$ & $0(0.0)$ & 0.16 \\
Bleeding & $1(1.6)$ & $1(1.6)$ & 1.00 \\
Cardiac tamponade & $0(0.0)$ & $1(1.6)$ & 0.32 \\
Total & $3(4.8)$ & $2(3.2)$ & 0.65 \\
\hline
\end{tabular}

Abbreviations as in Tables 1,2. discontinuation of DOACs was not inferior to CA without discontinuing VKA therapy.

Radiofrequency left atrial CA has a low risk of symptomatic cerebral ischemia, but is associated with a substantial risk of silent cerebral ischemia detected on magnetic resonance imaging. ${ }^{18}$ Gaita et al showed that independent risk factors for cerebral thromboembolism are the activated clotting time and electric or pharmacological cardioversion to sinus rhythm during the procedure. ${ }^{18}$

One of the reasons to continue anticoagulant therapy is 
to prevent any cessation in anticoagulant effect. In this study, the periprocedural activated clotting time was maintained at 300-350s and after the ablation was completed, in our strategy protamine sulfate was administered to reverse the effect of heparin as in the COMPARE trial. ${ }^{2}$ We observed spontaneous echo contrast after cardioversion in many cases, especially in patients with PeAF and LSAF, because "atrial kick" is poor despite sinus rhythm. Therefore, thrombus formation might occur shortly after AF ablation, but can be avoided by continuation of anticoagulation therapy.

The major concern of performing CA without discontinuation of anticoagulants is the risk of bleeding, as in intracerebral hemorrhage and cardiac tamponade. In this study, there was no significant difference between the 2 groups in bleeding events. Performing CA without discontinuation of DOACs is encouraged because of the existence of DOAC reversal agents, such as idarucizumab, in case of emergency major bleeding. Idarucizumab is a dabigatranspecific reversal agent that immediately and completely reverses the anticoagulant effect. ${ }^{\mathbf{1 9 , 2 0}}$

Our findings strongly supported that uninterrupted administration of all DOACs was not inferior to uninterrupted warfarin therapy, which is clinically significant. CA of AF can be performed without discontinuation of DOACs similar to the strategy with uninterrupted VKA therapy.

\section{Study Limitations}

This was a retrospective, observational, nonrandomized, single-center study and the results may not be generalizable. We also had to compare the 2 groups with propensity score matching, which decreased the number of entries in this study.

\section{Conclusions}

Performing CA of AF without discontinuation of DOACs is not inferior to CA without discontinuation of VKA therapy, with regard to ischemic or hemorrhagic complications in the periprocedural period.

\section{Disclosures}

The authors report no conflicts of interest related to this study.

\section{References}

1. Calkins H, Kuck KH, Cappato R, Brugada J, Camm AJ, Chen SA, et al. 2012 HRS/EHRA/ECAS expert consensus statement on catheter and surgical ablation of atrial fibrillation: Recommendations for patient selection, procedural techniques, patient management and follow-up, definitions, endpoints, and research trial design. Europace 2012; 14: 528-606.

2. Di Biase L, Burkhardt JD, Santangeli P, Mohanty P, Sanchez JE, Horton R, et al. Periprocedural stroke and bleeding complications in patients undergoing catheter ablation of atrial fibrillation with different anticoagulation management: Results from the role of coumadin in preventing thromboembolism in atrial fibrillation (AF) patients undergoing catheter ablation (COMPARE) randomized trial. Circulation 2014; 129: 2638-2644.

3. Page SP, Siddiqui MS, Finlay M, Hunter RJ, Abrams DJ, Dhinoja $\mathrm{M}$, et al. Catheter ablation for atrial fibrillation on uninterrupted warfarin: Can it be done without echo guidance? J Cardiovasc Electrophysiol 2011; 22: 265-270.

4. Calkins H, Willems S, Gerstenfeld EP, Verma A, Schilling R, Hohnloser SH, et al. Uninterrupted dabigatran versus warfarin for ablation in atrial fibrillation. N Engl J Med 2017; 376: 16271636.

5. Lakkireddy D, Reddy YM, Di Biase L, Vallakati A, Mansour MC, Santangeli P, et al. Feasibility and safety of uninterrupted rivaroxaban for periprocedural anticoagulation in patients undergoing radiofrequency ablation for atrial fibrillation: Results from a multicenter prospective registry. J Am Coll Cardiol 2014; 63: $982-988$.

6. Maddox W, Kay GN, Yamada T, Osorio J, Doppalapudi H, Plumb VJ, et al. Dabigatran versus warfarin therapy for uninterrupted oral anticoagulation during atrial fibrillation ablation. $J$ Cardiovasc Electrophysiol 2013; 24: 861-865.

7. Calkins H, Hindricks G, Cappato R, Kim YH, Saad EB, Aguinaga L, et al. $2017 \mathrm{HRS} / \mathrm{EHRA} / \mathrm{ECAS} / \mathrm{APHRS} / \mathrm{SOLAECE}$ expert consensus statement on catheter and surgical ablation of atrial fibrillation. Heart Rhythm 2017; 14: e275-e444.

8. Calkins H, Hindricks G, Cappato R, Kim YH, Saad EB, Aguinaga L, et al. $2017 \mathrm{HRS/EHRA/ECAS/APHRS/SOLAECE}$ expert consensus statement on catheter and surgical ablation of atrial fibrillation: Executive summary. Europace 2017; 20: $157-$ 208.

9. Briceno DF, Villablanca PA, Lupercio F, Kargoli F, Jagannath A, Londono A, et al. Clinical impact of heparin kinetics during catheter ablation of atrial fibrillation: Meta-analysis and metaregression. J Cardiovasc Electrophysiol 2016; 27: 683-693.

10. Di Biase L, Elayi CS, Fahmy TS, Martin DO, Ching CK, Barrett $\mathrm{C}$, et al. Atrial fibrillation ablation strategies for paroxysmal patients: Randomized comparison between different techniques. Circ Arrhythm Electrophysiol 2009; 2: 113-119.

11. Mehran R, Rao SV, Bhatt DL, Gibson CM, Caixeta A, Eikelboom $\mathrm{J}$, et al. Standardized bleeding definitions for cardiovascular clinical trials: A consensus report from the Bleeding Academic Research Consortium. Circulation 2011; 123: 2736-2747.

12. Ndrepepa G, Schuster T, Hadamitzky M, Byrne RA, Mehilli J, Neumann FJ, et al. Validation of the Bleeding Academic Research Consortium definition of bleeding in patients with coronary artery disease undergoing percutaneous coronary intervention. Circulation 2012; 125: 1424-1431.

13. Shah RR, Pillai A, Schafer P, Meggo D, McElderry T, Plumb V, et al. Safety and efficacy of uninterrupted apixaban therapy versus warfarin during atrial fibrillation ablation. Am J Cardiol 2017; 120: $404-407$.

14. Nagao T, Inden Y, Shimano M, Fujita M, Yanagisawa S, Kato $\mathrm{H}$, et al. Feasibility and safety of uninterrupted dabigatran therapy in patients undergoing ablation for atrial fibrillation. Intern Med 2015; 54: 1167-1173.

15. Lakkireddy D, Reddy YM, Di Biase L, Vanga SR, Santangeli P, Swarup V, et al. Feasibility and safety of dabigatran versus warfarin for periprocedural anticoagulation in patients undergoing radiofrequency ablation for atrial fibrillation: Results from a multicenter prospective registry. J Am Coll Cardiol 2012; 59: $1168-1174$

16. Kim JS, She F, Jongnarangsin K, Chugh A, Latchamsetty R, Ghanbari $\mathrm{H}$, et al. Dabigatran vs. warfarin for radiofrequency catheter ablation of atrial fibrillation. Heart Rhythm 2013; 10: $483-489$.

17. Nin T, Sairaku A, Yoshida Y, Kamiya H, Tatematsu Y, Nanasato M, et al. A randomized controlled trial of dabigatran versus warfarin for periablation anticoagulation in patients undergoing ablation of atrial fibrillation. Pacing Clin Electrophysiol 2013; 36: 172-179.

18. Gaita F, Caponi D, Pianelli M, Scaglione M, Toso E, Cesarani F, et al. Radiofrequency catheter ablation of atrial fibrillation: A cause of silent thromboembolism? Magnetic resonance imaging assessment of cerebral thromboembolism in patients undergoing ablation of atrial fibrillation. Circulation 2010; 122: 1667-1673.

19. Kirchhof P, Benussi S, Kotecha D, Ahlsson A, Atar D, Casadei $\mathrm{B}$, et al. 2016 ESC guidelines for the management of atrial fibrillation developed in collaboration with EACTS. Eur Heart J 2016; 37: 2893-2962.

20. Kirchhof P, Benussi S, Kotecha D, Ahlsson A, Atar D, Casadei $\mathrm{B}$, et al. 2016 ESC guidelines for the management of atrial fibrillation developed in collaboration with EACTS. Europace 2016; 18: $1609-1678$

\section{Supplementary Files}

\section{Supplementary File 1}

Table S1. Hemorrhagic complication

Please find supplementary file(s);

http://dx.doi.org/10.1253/circj.CJ-17-1114 